Supporting Information

\title{
Active Micromotor Systems Built from Passive Particles with Biomimetic Predator- Prey Interactions
}

Fangzhi Mou, Xiaofeng Li, Qi Xie, Jianhua Zhang, Kang Xiong, Leilei Xu and

Jianguo Guan*

State Key Laboratory of Advanced Technology for Materials Synthesis and

Processing, International School of Materials Science and Engineering, Wuhan

University of Technology, 122 Luoshi Road, Wuhan 430070 (P.R. China)

KEYWORDS. Micro/nanomotors, collective behaviors, active systems, predator-prey behaviors, interactions, diffusiophoresis 


\section{Supporting Figures}

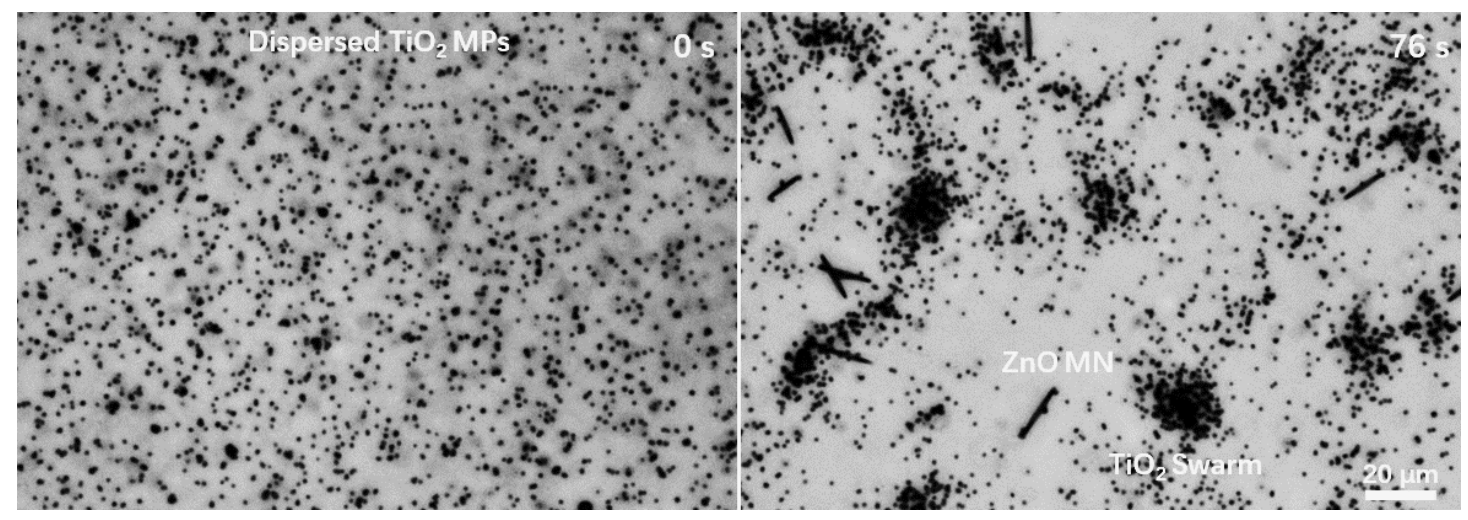

Figure S1. Time-lapse microscopic images depicting the clustering behavior of the $\mathrm{TiO}_{2} \mathrm{MPs}$ when they are mixed with $\mathrm{ZnO}$ MNs.

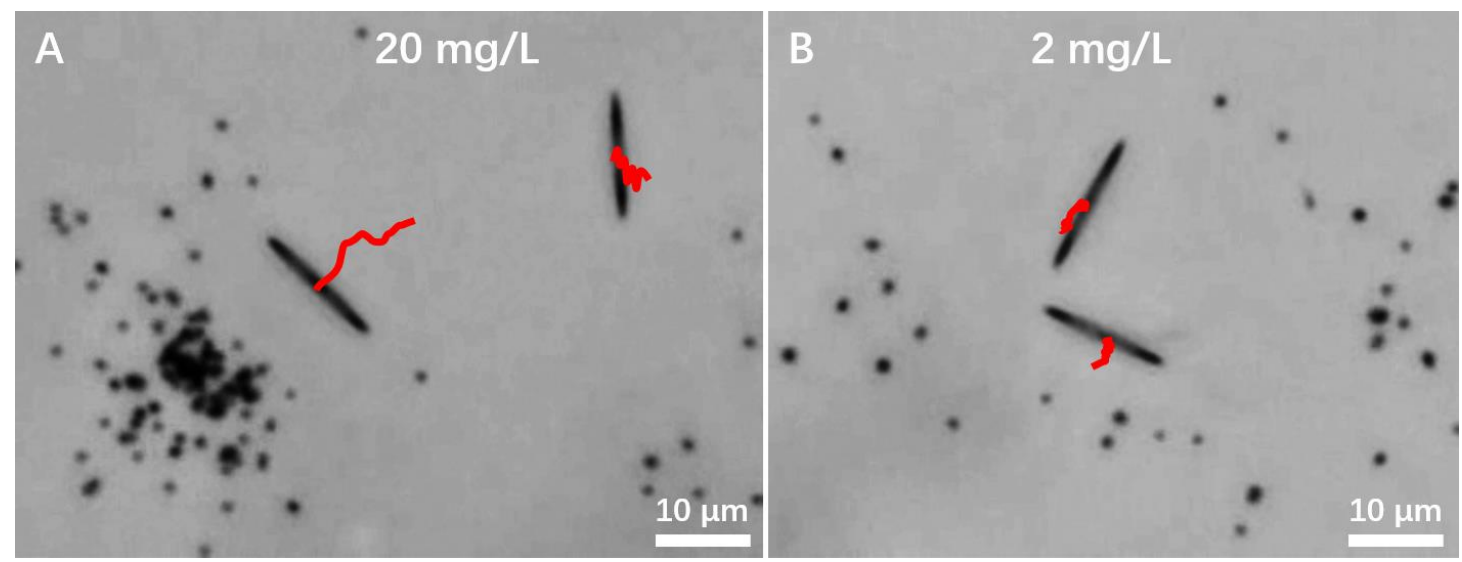

Figure S2. The trajectories of ZnO MNs in $30 \mathrm{~s}$ at $C_{\mathrm{T}}$ of (A) 20 and (B) $2 \mathrm{mg} / \mathrm{L}$ in water, respectively. 


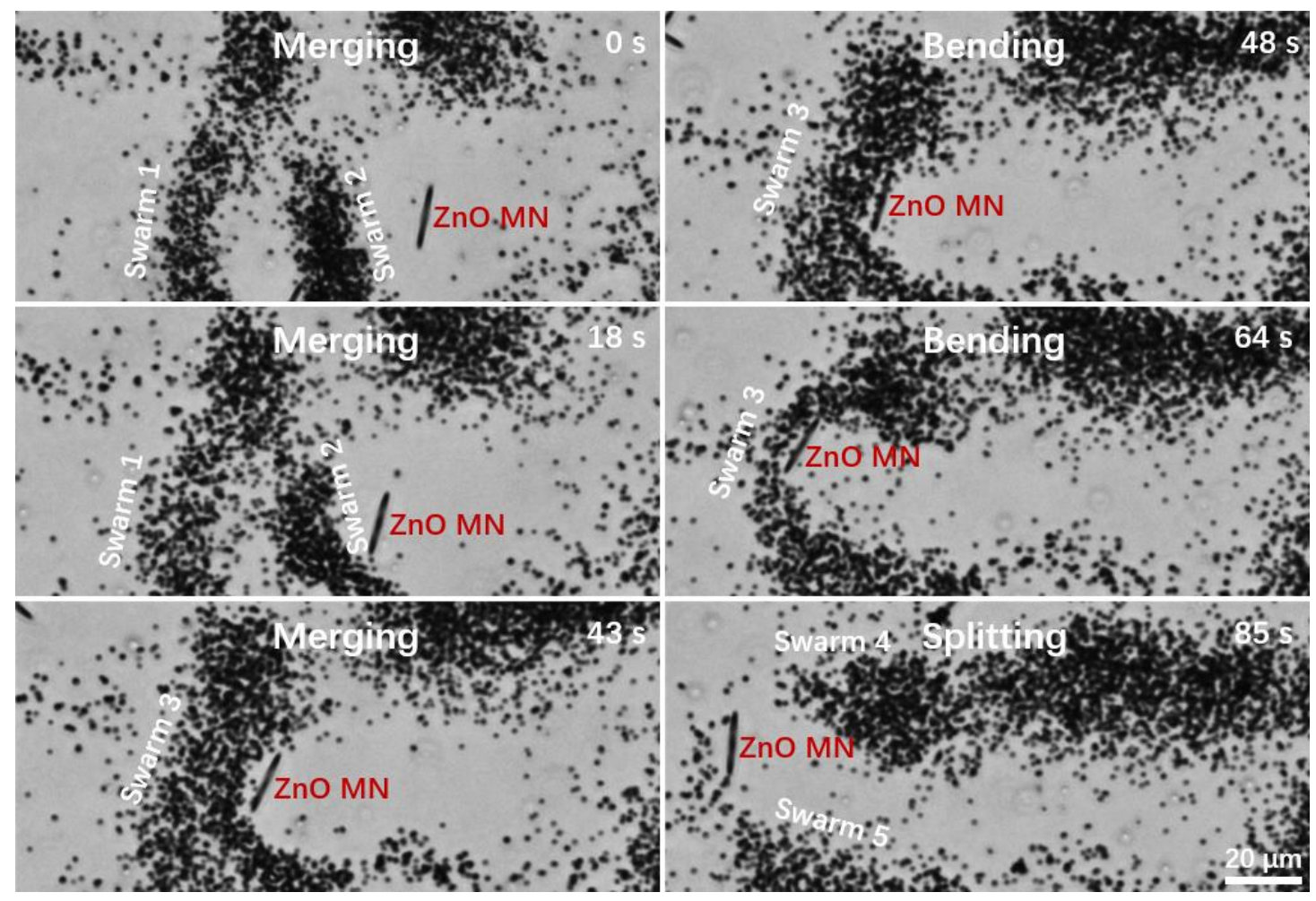

Figure S3. Time-lapse microscopic images representing the dynamic group reconfigurations of swarming $\mathrm{TiO}_{2}$ MPs in response to an approaching ZnO MN, including merging (0-43 s), bending (43-64 s) and splitting (64-85 s). In 0 - 43 s, Swarm 1 merges with Swarm 2 into Swarm 3 when $\mathrm{TiO}_{2}$ MPs in Swarm 2 escape from the $\mathrm{ZnO} \mathrm{MN}$. Then, when the $\mathrm{ZnO} \mathrm{MN}$ moves forward continuously, Swarm 3 bends into a cordon swarm in 43 - 64 s. Swarm 3 further splits into two swarms (Swarm 4 and 5) when the ZnO MN passes through it (64 - 85 s).

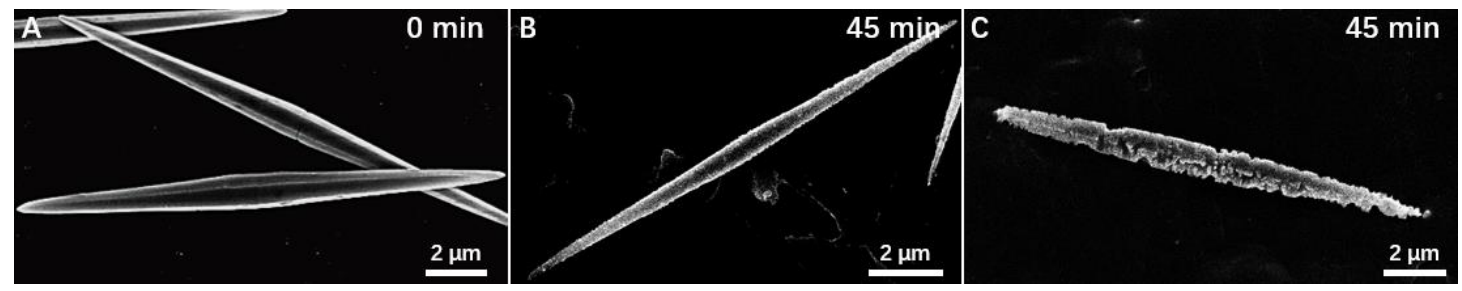

Figure S4. SEM images of the as-prepared $\mathrm{ZnO} \mathrm{MNs}$ (A) and those after soaking in (B) the deionized water and $(\mathrm{C})$ in the water suspension of $\mathrm{TiO}_{2} \mathrm{MPs}$ for $45 \mathrm{~min}$.

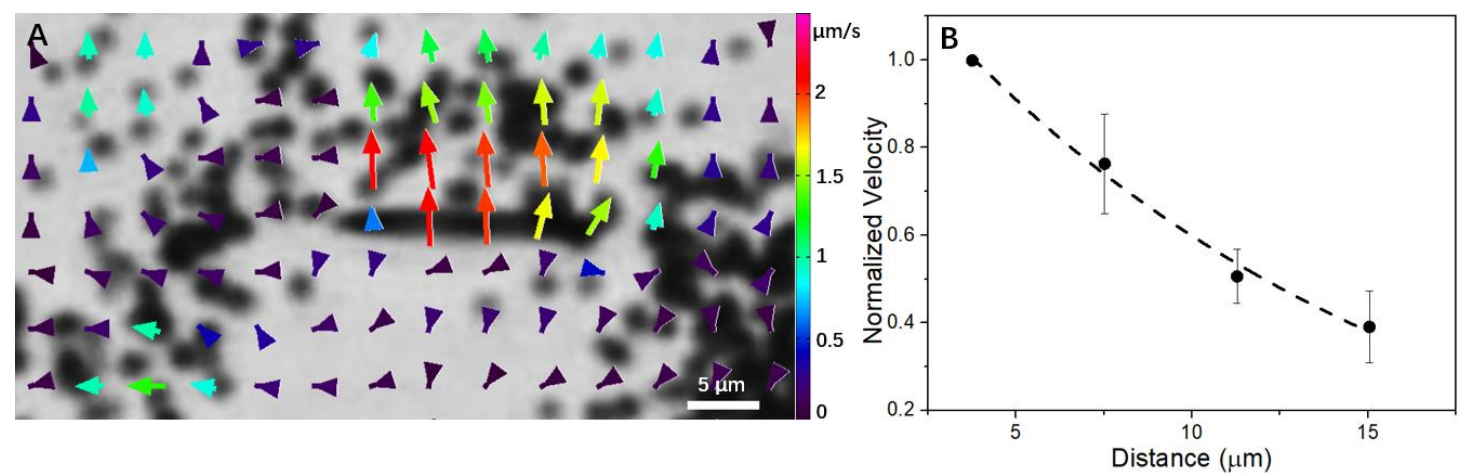

Figure S5. (A) The instantaneous velocity vectors of $\mathrm{TiO}_{2} \mathrm{MPs}$ around a $\mathrm{ZnO} \mathrm{MN}$. (B) The normalized velocity of the $\mathrm{TiO}_{2}$ MPs depending on their distance to the long axis of the $\mathrm{ZnO} \mathrm{MN}$. 


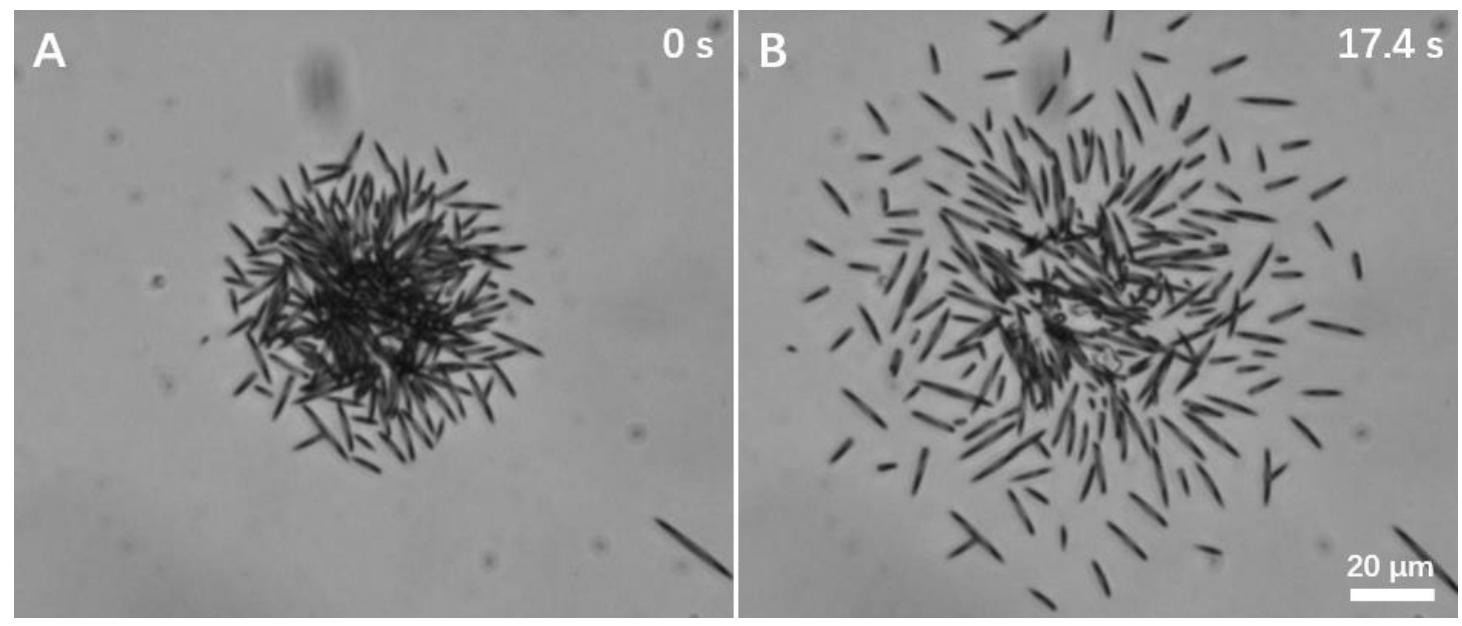

Figure S6. Time-lapse microscopic images representing the exclusion behavior of ZnO MNs. (A) the aggregated $\mathrm{ZnO} \mathrm{MNs}$ by the NIR-light-induced convection flow in water $(0 \mathrm{~s})$. (B) the scattered $\mathrm{ZnO} \mathrm{MNs}$ at $17.4 \mathrm{~s}$ after NIR light is off.

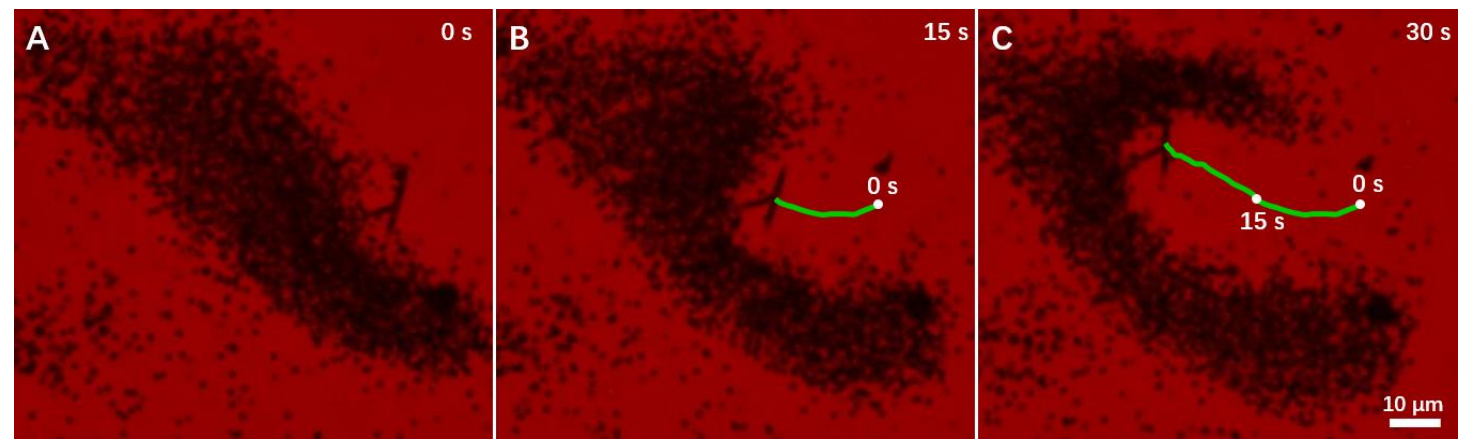

Figure S7. Time-lapse microscopic images depicting the motion of a $\mathrm{ZnO} \mathrm{MN}$ towards swarming $\mathrm{TiO}_{2}$ MPs in the low-energy red light $\left(I=20.2 \mathrm{~mW} / \mathrm{cm}^{2}\right)$.

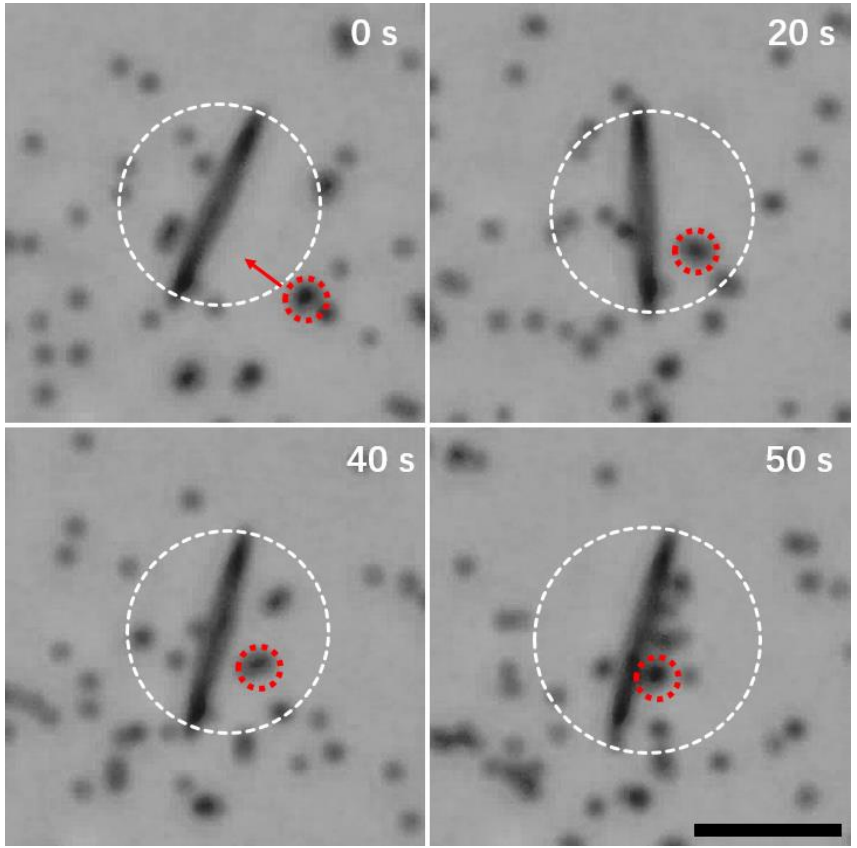

Figure S8. Time-lapse microscopic images depicting the motion of $\mathrm{TiO}_{2} \mathrm{MPs}_{\text {s }}$ towards a $\mathrm{ZnO} \mathrm{MN}$ on the positive-charge modified glass slide. 


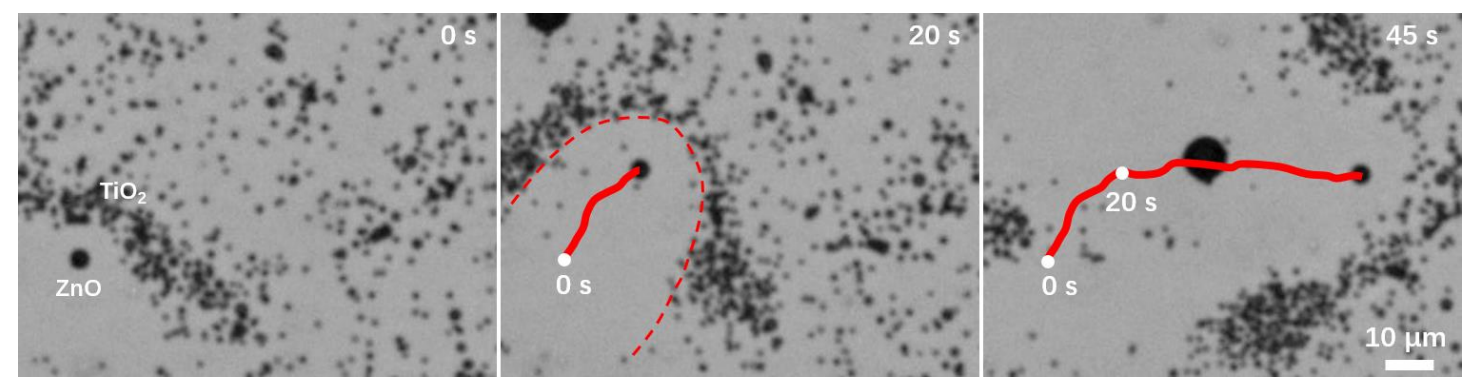

Figure S9. Time-lapse microscopic images showing the "preytaxis" of a spherical ZnO MP towards swarming $\mathrm{TiO}_{2}$ MPs. 


\section{Supporting Videos}

Video S1. Spontaneous clustering of $\mathrm{TiO}_{2} \mathrm{MPs}$ in water.

Video S2. Motions of $\mathrm{ZnO}$ MNs and $\mathrm{TiO}_{2}$ MPs in water before and after being mixed.

Video S3. Dynamic group reconfigurations of the swarming $\mathrm{TiO}_{2} \mathrm{MPs}$ and reciprocating motions of the $\mathrm{ZnO}$ MNs towards the swarming $\mathrm{TiO}_{2} \mathrm{MPs}$.

Video S4. The attraction of a swarm of $\mathrm{TiO}_{2}$ MPs to adjacent passive $\mathrm{SiO}_{2}(-)$ and the PS(+) TPs. Video S5. The repulsion of a $\mathrm{ZnO} \mathrm{MN}$ to adjacent passive $\mathrm{SiO}_{2}(-)$ and the $\mathrm{PS}(+)$ TPs.

Video S6. The motions of the $\mathrm{ZnO} \mathrm{MNs}$ and $\mathrm{TiO}_{2} \mathrm{MPs}$ in low-energy red light and on the positivecharge modified glass substrate, respectively, and the chasing-escaping motions of the spherical $\mathrm{ZnO}$ MPs and $\mathrm{TiO}_{2}$ MPs.

Video S7. The chasing-escaping motions in $\mathrm{Ag}_{3} \mathrm{PO}_{4}-\mathrm{TiO}_{2}$ and $\mathrm{ZnO}-\mathrm{AgBr}$ binary micromotor systems. 


\section{Governing equations for numerical simulations.}

The distribution of various ions $\left(\mathrm{Zn}^{2+}, \mathrm{H}^{+}\right.$and $\left.\mathrm{OH}^{-}\right)$originates from the fluxes of the ions from the surface of the particles ( $\mathrm{ZnO} \mathrm{MNs}$ and $\left.\mathrm{TiO}_{2} \mathrm{MPs}\right)$, and is further affected by the diffusion, convection and migration of ions (Equation 1). In our model, this is solved with the conservation equation (Equation 2) at steady state.

$$
\begin{aligned}
& \boldsymbol{J}_{i}=\boldsymbol{u} c_{i}-D_{i} \boldsymbol{\nabla} c_{i}-\frac{z_{i} F D_{i} c_{i} \nabla \varphi}{\mathrm{RT}} \\
& \boldsymbol{\nabla} \cdot \boldsymbol{J}_{i}=\mathbf{0}=\boldsymbol{u} \cdot \boldsymbol{\nabla} c_{i}-D_{i} \boldsymbol{\nabla}^{2} c_{i}-\frac{z_{i} F D_{i} \boldsymbol{\nabla} \cdot\left(c_{i} \nabla \varphi\right)}{\mathrm{RT}}
\end{aligned}
$$

Where $\boldsymbol{u}$ is the fluid velocity, $F$ is the Faraday constant, $\varphi$ is the electrostatic potential, $\mathrm{R}$ is the gas constant, $\mathrm{T}$ is the absolute temperature, and $c_{i}, D_{i}, z_{i}$ are the concentration, diffusion coefficient, and charge of species $i$, respectively.

The electric potential $(\varphi)$ in Equation 1 is calculated using the Poisson equation,

$-\varepsilon_{0} \varepsilon_{r} \nabla^{2} \varphi=\rho_{e}=F\left(z_{+} c_{+}+z_{-} c_{-}\right)$

where $\rho_{e}$ is the volumetric charge density, $z_{+}$and $z_{-}$are the charges of the cations and the anions, $c_{+}$and $c_{-}$are the concentrations of the cations and the anions, $\varepsilon_{0}$ is the permittivity of the vacuum, and $\varepsilon_{r}$ is the relative permittivity of the fluid media, respectively.

The inertial effect is neglected in the present study because of a very small Reynolds number. Thus, the flow field is governed by the Stokes equations,

$-\nabla p+\mu \nabla^{2} \boldsymbol{u}=0$

and the continuity equation for the incompressible fluid,

$\nabla \cdot \boldsymbol{u}=0$

In these equations, $\boldsymbol{u}$ is the fluid velocity vector, and $p$ is the pressure. The initial values of the flow velocity and the pressure are all zero. The electroosmotic flow boundary conditions are as following:

On the particle surface, $\boldsymbol{u}=\frac{\varepsilon_{0} \varepsilon_{r} \zeta_{p}}{\mu}(\mathbf{I}-\mathbf{n n}) \cdot \nabla \varphi$

On the substrate surface, $\boldsymbol{u}=\frac{\varepsilon_{0} \varepsilon_{r} \zeta_{w}}{\mu}(\mathbf{I}-\mathbf{n n}) \cdot \nabla \varphi$

In this equation, $\zeta_{p}$ and $\zeta_{w}$ are the Zeta potential of the particles ( $\mathrm{ZnO}$ MNs and $\mathrm{TiO}_{2} \mathrm{MPs}$ ) and the wall (substrate), respectively. The quantity $(\mathbf{I}-\mathbf{n n})$ defines the electric field tangential to the charged surface, with $\mathbf{I}$ denoting the second-order unit tensor. 